\title{
Erratum to: On flowshop scheduling problems with the aging effect and resource allocation
}

\author{
Radosław Rudek $^{1}$ • Agnieszka Wielgus ${ }^{2}$
}

Published online: 19 October 2015

(C) Springer-Verlag London 2015

\section{Erratum to: Int J Adv Manuf Technol (2012) 62:135-145 DOI 10.1007/s00170-011-3809-1}

In our paper Rudek and Rudek (Int J Adv Manuf Technol 62:135-145, 2012), we have published incomplete version of a polynomial time algorithm for the makespan minimization flowshop scheduling problem with the aging effect and the allocation of additional resource. Here, we provide the previously accidentally deleted lines of the algorithm, which make it complete and correct.

\section{Problem formulation}

In our paper [1], we analyzed inter alia the makespan minimization flowshop problem with the aging effect and the resource allocation. Let us briefly recall it.

There are given a set of $n$ jobs and two machines. Each job is available at time zero and has to be processed by each

The online version of the original article can be found at http://dx. doi.org/10.1007/s00170-011-3809-1.

Radosław Rudek

radoslaw.rudek@ue.wroc.pl

Agnieszka Wielgus

agnieszka.wielgus@pwr.wroc.pl

1 Wrocław University of Economics, Wrocław, Poland

2 Wrocław University of Technology, Wrocław, Poland of the machines in the same order and no preemption is allowed. The processing time of job $j$ on the first machine depends on its position $v$ in a sequence and on the allocated resource $u_{j}$ as follows $p_{j}^{(1)}\left(v, u_{j}\right)=a_{j}^{(1)}+b^{(1)} v-\gamma u_{j}$, where $a_{j}^{(1)}$ is the normal processing time of $j, b^{(1)}$ is an aging ratio and $\gamma$ is the resource ratio that denotes the decreasing of a job processing time depending on the amount of allocated resource $u_{j}$. The processing time of job $j$ on the second machine is equal to its normal processing time $p_{j}^{(2)}=a_{j}^{(2)}$. It is also assumed that $0 \leq u_{j} \leq \bar{u}_{j}$ and $\sum_{j=1}^{n} u_{j} \leq \hat{U}$, where $\bar{u}_{j}$ is the maximal amount of the resource that can be allocated to job $j$ and $\hat{U}$ is a global restriction on the total resource consumption. The objective is to find such a permutation of jobs $\pi$ and the resource allocation to jobs $\mathbf{u}$ that minimize the makespan $C_{\max }(\pi, \mathbf{u})$; in the further part, $\pi(i)$ denotes a job scheduled in position $i$ in permutation $\pi$.

\section{Algorithm}

In our paper [1], we provided Property 8 for the considered problem as follows:

Property 8 [1] The problem $F 2 \mid p_{j}^{(1)}\left(v, u_{j}\right)=a_{j}^{(1)}+b^{(1)} v-$ $\gamma u_{j}, p_{j}^{(2)}\left(v, u_{j}\right)=a^{(2)}, \sum u_{j} \leq \hat{U} \mid C_{\max }$ can be solved optimally in $O(n \log n)$ steps according to Algorithm 1.

However, the related algorithm was published without few important lines. Their obvious lack clearly follows from the proof of Property 8 . Therefore, we present the complete version of Algorithm 1 with the previously accidentally deleted lines, which are denoted by '*'. 


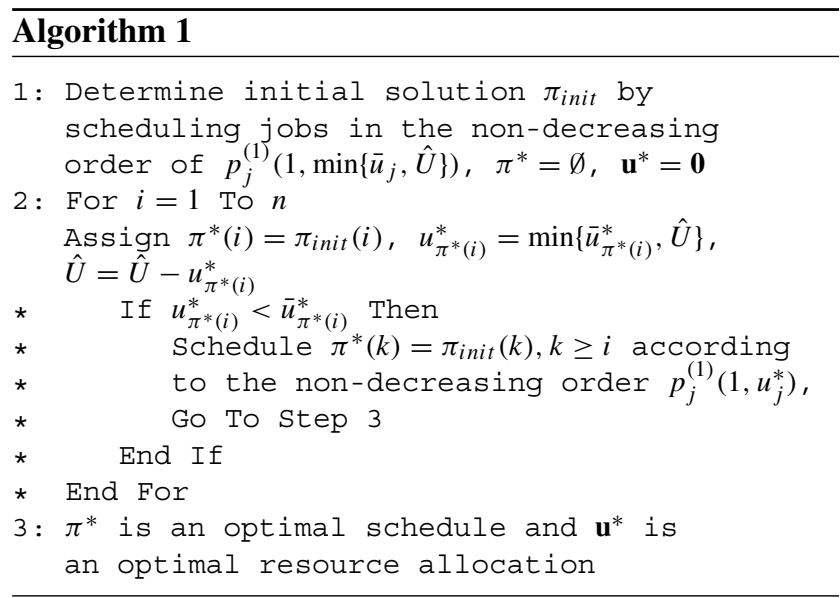

Since, we have been alerted that the proof of Property 8 may not be clear, therefore, we will also give some additional comments.

In our paper [1], we proved that there exists an optimal solution, where the resource is allocated according to the maximum possible amount (the first part of the proof) taking into consideration that jobs are scheduled according to the non-decreasing sequence of $p_{j}^{(1)}\left(1, u_{j}\right)$ (the second part). Namely, it was shown that the following difference [1] (page 142 , col. 2, line -1) $p_{\pi(i)}^{(1)}\left(i, u_{\pi(i)}^{\prime(1)}\right)-p_{\pi^{\prime}(i)}^{(1)}\left(i, u_{\pi^{\prime}(i)}^{(1)}\right.$ ) (which can also be written as $p_{\pi(i)}^{(1)}\left(1,{u_{\pi(i)}^{\prime}}_{(1)}\right)-p_{\pi^{\prime}(i)}^{(1)}\left(1,{u^{\prime}}_{\pi^{\prime}(i)}^{(1)}\right)$, since the difference is position independent) is nonnegative, thereby the optimal solution has to hold the non-decreasing sequence of $p_{j}^{(1)}\left(1, u_{j}\right)$.

The algorithm starts with the initial sequence of jobs $\pi_{i n i t}$, which have the resource allocated in the maximum possible amount (i.e., $\left.p_{j}^{(1)}\left(1, \min \left\{\bar{u}_{j}, \hat{U}\right\}\right)\right)$ and it is obtained in Step 1. Obviously, if the resource is depleted $\left(u_{\pi^{*}(i)}^{*}<\right.$ $\bar{u}_{\pi^{*}(i)}^{*}$ ), then the initial sequence $\pi_{\text {init }}$ (constructed on the basis of $\left.p_{j}^{(1)}\left(1, \min \left\{\bar{u}_{j}, \hat{U}\right\}\right)\right)$ may be no longer optimal, since remaining jobs (in positions $i, \ldots, n$ ) may not hold the non-decreasing sequence according to $p_{j}^{(1)}\left(1, u_{j}\right)$. Therefore, the first job holding $u_{\pi^{*}(i)}^{*}<\bar{u}_{\pi^{*}(i)}^{*}$ (i.e., for which the resource cannot be allocated to the maximum possible amount) and next jobs have to be rescheduled according to the non-decreasing order of $p_{j}^{(1)}\left(1, u_{j}\right)$, which concerns jobs in positions $k=i, \ldots, n$ in $\pi_{i n i t}$. After that the algorithm terminates. This part is included in Step 2. Thus, the solution constructed in this way (the permutation of jobs $\pi^{*}$ and the allocation of the resource $\left.\mathbf{u}^{*}\right)$ is optimal and the time complexity is $O(n \log n)$.

Additionally, we have also found obvious typos in [1] that clearly did not correspond to its content, i.e., page 142, col. 1 , line -22: $p_{j}^{(1)}\left(1, \min \left\{\bar{u}_{j}, \hat{U}\right\}\right) \rightarrow p_{j}^{(1)}\left(1, \min \left\{u_{j}, \hat{U}\right\}\right)$; page 142, col. 2, line 24 and page 143, col. 1, line 1: $p_{\pi(i)}^{(1)}\left(1, \min \left\{\bar{u}_{\pi(i)}, \hat{U}\right\}\right)<p_{\pi(i+1)}^{(1)}\left(1, \min \left\{\bar{u}_{\pi(i+1)}, \hat{U}\right\}\right) \rightarrow$ $p_{\pi(i)}^{(1)}\left(1, \min \left\{u_{\pi(i)}, \hat{U}\right\}\right)>p_{\pi(i+1)}^{(1)}\left(1, \min \left\{u_{\pi(i+1)}, \hat{U}\right\}\right) ;$ where " $\rightarrow$ " means "should be replaced by".

Funding The research elucidation presented in this paper has been supported by the Polish Ministry of Science and Higher Education under Iuventus Plus Programme (No. IP2014 040673).

\section{References}

1. Rudek A, Rudek R (2012) On flowshop scheduling problems with the aging effect and resource allocation. Int J Adv Manuf Technol 62:135-145 